\title{
A genome-wide analysis of simple sequence repeats in maize and the development of polymorphism markers from next-generation sequence data
}

Jingtao $\mathrm{Qu}^{1+}$ and Jian $\mathrm{Liu}^{1,2^{*}+}$

\begin{abstract}
Background: Maize (Zea mays ssp. mays L.), as the most important plant for staple food of several million people, animal feed and bioenergy productions, is widely cultivated around the world. Simple sequence repeats (SSRs) are widely used as molecular markers in maize genetics and breeding, but only two thousands pairs of SSRs have been published currently, which hardly satisfies for the increasing needs of geneticists and breeders. Furthermore, the increasing studies have revealed that SSRs also play a vital role in functional regulation and evolution. It is fortunate that the development of sequencing technology and bio-software provides the basis for characterization and development of SSRs in maize.

Results: In this study, MISA was applied to identify overall 179,681 SSRs in maize reference genome B73, with an average distance of $11.46 \mathrm{Kbp}$. Their distributions within the genome in different regions were non-random, and the density followed in a descending order of UTR, promotor, intron, intergenic and CDS. Meanwhile, 82,694 (46.02\%) SSRs with unique flanking sequences were selected, and then applied to analyze the polymorphism of next-generation sequencing data from 345 maize inbred lines and data from maize reference genome B73. There were 58,946 SSRs with length information results in ten or more than ten genomes, accounting for $71.28 \%$ of SSRs with unique flanking sequences, while 55,621 SSRs had polymorphism, with an average PIC value of 0.498. 250 pairs of SSR primers in different genomic regions covering all maize chromosomes were randomly chosen for the experimental validation, with an average PIC value of 0.63 in 11 elite maize inbred lines.
\end{abstract}

Conclusions: Our work provided insight into the non-random distribution spatterns and compositions of SSRs in different regions of maize genome, and also developed more polymorphic SSR markers using next-generation sequencing reads. The genome-wide SSRs polymorphism markers could be useful for genetic analysis and marker-assisted selection in breeding practice, and it was also proved to be high efficient for molecular marker development via next-generation sequencing reads.

Keywords: Maize, Simple sequence repeat (SSR), Genome, Next-generation sequence, Polymorphism

\footnotetext{
*Correspondence: ljian79@hotmail.com

${ }^{\dagger}$ Equal contributors

${ }^{1}$ Maize Research Institute, Sichuan Agricultural University, Chengdu, Sichuan 611130, China

${ }^{2}$ Key Laboratory of Biology and Genetic Improvement of Maize in Southwest Region, Ministry of Agriculture, Chengdu, China
} 


\section{Background}

Simple sequence repeats (SSRs) or microsatellites were tandemly arranged repeats of short DNA motifs (1-6 nucleotides long), which extensively distributed in eukaryotes including the plants, animals and microorganisms, as well as in some prokaryotes [1]. SSRs were commonly regarded as genomic "junk" with no significant role as genomic information in a long time until the more utilizing of SSR repeat-number variation and accumulating evidence to support the hypothesis that SSRs could play a positive role in adaptive evolution [2-4]. For assaying genetic variation, SSRs markers based on the repeat number variation were showing significant advantages over the variety of other molecular markers, including restriction fragment length polymorphisms (RFLPs), random amplification of polymorphic DNA (RAPD), and amplified fragment length polymorphisms (AFLPs) [5-7]. As a codominant marker, SSRs have proven to be highly polymorphic, easily reproducible, low costing, facility amplified, and not specifically linked to gene loci of immediate interest [8]. Just for these reasons, SSRs markers turned out to be ideal molecular markers which were widely used in genetic and evolution researches, even as the preferred marker system for many breeding applications. As the development of molecular technology and bioinformatics, increasingly more SSRs with possible functions have been found and characterized, and multiple studies have proved the functional relevance of a significant number of SSRs [2-4,9]. The persistence of intragenic repeats in genomes suggested that there was a compensating benefit [10]. In Mycoplasma, a variety of SSRs repeats acted as contingency loci by modulating gene expression or facilitating genome rearrangements via recombination, affecting protein structure and possibly protein-protein interactions, even contributing to the organization of the DNA molecule in cells [9]. Additionally, genes containing intragenic repeats encoded cell-wall proteins in the genome of Saccharomyces cerevisiae, which revealed that the variation in intragenic repeat number provided the functional diversity of cell surface antigens allowed rapid adaptation to the environment and elusion of the host immune system in fungi and other pathogens [10]. In humans, allelic differences of SSR repeats numbers were known to cause a wide range of hereditary disorders and disease susceptibilities, such as the 'triplet repeat diseases' $[2,11]$. The presence of SSRs in transcripts of genes in plant species suggested that it might have a role in gene expression and regulation [5,12-14]. The repetitive GCC triplets in the 5'UTRs of ribosomal protein transcripts in maize were believed to influence both gene expression and translation efficiency for the regulation of fertilization [14]. Similarly, SSRs located in the 5'UTR of rice waxy gene were correlated with amylase content [13].

Maize (Zea mays ssp. mays L.), as the most important plant for staple food of several million people, animal feed and bioenergy productions, is widely cultivated around the world. Therefore, it now poses a serious threat to maize production including yield persistently increasing, quality enhancement, disease and insect damage intensifying and extreme environments. In this situation, it's urgently needed to strengthen genetic researches and improve breeding efficiency in maize. As a polymerase chain reaction (PCR) is based on efficient molecular markers, SSRs play an important role in maize genetic researches and breeding for a long time. So far, almost two thousands pairs of SSR primers have been published, but they hardly satisfy for the increasing needs of geneticists and breeders. Additionally, the development of SSRs is considerably costly and time consuming through the traditional approaches, but it is fortunate that highthroughput sequencing technologies allow the isolation and development of SSR for more efficient genetic research with high abundance. A series of software for scanning SSRs in the genome have been developed by computational biologists, such as MISA and SSRIT. Benefit from these achievements, the distribution and variation of SSRs frequency were revealed by more researches across species [15-18]. Taking the sequence of maize inbred line B73 as a reference genome, extensive researches are dedicated to structure variation of the genome and transposon identifications [19-21]. With the development of next-generation sequencing technologies, many more cultivars have been analyzed by de novo sequencing due to its dramatically low cost and short time. The de novo sequencing data from 278 and 86 maize inbred lines were published by Jinsheng Lai and JerMing Chia respectively in August 2012 [22,23], but the distribution and frequency of SSRs in maize genome have not been investigated.

Hence, the goal of this study was to reveal the patterns of SSR distribution in maize genome and explore the database of maize SSR markers to saturate the genetic linkage map. Meanwhile, SSR polymorphism markers were filtered by comparing SSRs in the sequencing data from 345 maize inbred lines and maize reference genome B73. The genome-wide SSRs polymorphism markers could be useful for genetic analysis and marker-assisted selection in breeding practice.

\section{Results}

Identification and distribution of SSRs in maize genome

A total of 179,681 SSRs were identified on the whole 10 chromosomes, and the average distance between repeat units varied from $11.12 \mathrm{~Kb}$ (Chromosome 6) to $11.89 \mathrm{~Kb}$ (Chromosome 4), with an average of $11.46 \mathrm{~Kb}$. The detailed information of identified SSRs in maize was summarized in Additional file 1. For the total number of SSRs on each chromosome, Chromosome 1 harbored the maximum number of SSRs $(26,718)$, while Chromosome 10 had 
the minimum $(13,179)$, which implied that the number of SSRs on chromosome could be positively correlated with chromosome length.

SSRs were distributed in different genomic regions, including promoters, 5'UTR (untranslated region), 3'UTR, CDS (coding sequence), intron, and intergenic regions. As shown in Table 1, most abundance of SSRs was located in the intergenic region (77.25\%), while $1.86 \%$ was located in the CDS regions. The density of SSRs in different areas of genome varied and followed in a descending order of 5'UTR, 3'UTR, promotor, intron, intergenic and CDS.

Due to over $85 \%$ of the maize B73 genome $(2.4 \mathrm{~Gb})$ consisted of repetitive DNA, it was necessary to explore the specific SSRs for further researches and applications [19]. There were 82,694 SSRs with unique flanking sequences (unique SSRs), accounting for $46.02 \%$ of the entire number of SSRs. Consistent with the total SSRs in genome, the proportion of unique SSRs was located in the intergenic region ranking the highest (56.76\%), while $43.24 \%$ of the unique SSRs were found in genes (Table 1). Different from the distribution of overall SSRs in genome, although there were an abundance of unique SSRs in intergenic region as well, the density of which was extremely low, with an average distance of $38.23 \mathrm{~Kb}$ between two specific loci, far below the density of other regions. In addition, the details of SSRs in different regions were investigated. The result indicated that GC content of SSRs in CDS was up to $80.12 \%$, which was significant higher than any other regions of the genome. Meanwhile, the average sequence length of SSRs in CDS was much longer as well (Table 1).
Frequencies, repeat sequence length, motif repeats and distribution of different SSR repeat types in maize

The result of detected SSRs by MISA program contained perfect SSRs, compound SSRs and imperfect repeats. Among these three types, perfect SSRs were more abundant with a total number of 166,691 (92.77\%). 2,149 (1.20\%) SSRs were compound SSRs, containing two or more adjacent motifs in repeats. The imperfect SSRs accounted for $6.03 \%(10,841)$, and the repeats of which were interrupted by short tandems. Among the perfect repeats, the most common were MNRs (40.21\%), followed by DNRs (29.97\%) and TNRs (20.44\%). The occurrences of these three SSR types with different repeat unit sizes, a total of 162,826 collectively accounted for $90.62 \%$ of the total SSRs. The remaining repeat units, including TTRs, PNRs and HNRs, were made up for $2.15 \%(3,865)$ of the total SSRs. The proportion of different SSR types was listed in Table 2.

To assess the contribution of repeat sequence length to SSR abundance, the average length for different types of SSRs was calculated. The total average length of the overall SSRs was 20.34 bp. With regard to different kinds of SSRs, the average length of perfect SSRs, compound SSRs and imperfect SSRs was $15.41 \mathrm{bp}, 43.48 \mathrm{bp}$ and 91.66 bp respectively. For perfect SSRs, accounting for the majority of the total SSRs, the length of different repeat unit size varied from 10 bp to $1926 \mathrm{bp}$ and $91.31 \%$ of the total SSRs ranged from $10 \mathrm{bp}$ to $50 \mathrm{bp}$. (AGT) $)_{642}$, identified on chromosome 10 (63,833,394-63,835,319 bp), was considered to be the maximum of SSR length (1,926 bp) for perfect SSRs. The average length of HNRs reached $32.54 \mathrm{bp}$, which was significant longer than the remaining

Table 1 The distribution of SSRs in different areas of genome

\begin{tabular}{|c|c|c|c|c|c|c|c|c|c|c|c|c|c|c|c|}
\hline \multirow[t]{2}{*}{ Genome regions } & \multicolumn{4}{|c|}{ Overall SSRs $^{a}$} & \multicolumn{5}{|c|}{ Unique SSRs $^{\mathbf{b}}$} & \multicolumn{5}{|c|}{ Unique SSRs with polymorphisms ${ }^{c}$} & \multirow[t]{2}{*}{$\operatorname{Rate}^{\mathrm{i}}(\%)$} \\
\hline & Count & $\begin{array}{c}\text { Interval }^{d} \\
\text { (Kbp) }\end{array}$ & $\begin{array}{l}\text { Length } \\
\text { (bp) }\end{array}$ & $\begin{array}{l}G^{f} \\
(\%)\end{array}$ & Count & $\begin{array}{c}\text { Interval }^{\mathrm{d}} \\
\text { (Kbp) }\end{array}$ & $\begin{array}{l}\text { Length } \\
\text { (bp) }\end{array}$ & $\begin{array}{l}\text { GC } \\
(\%)\end{array}$ & $\begin{array}{c}\text { Rate }^{g} \\
(\%)\end{array}$ & Count & $\begin{array}{c}\text { Interval }^{d} \\
\text { (Kbp) }\end{array}$ & $\begin{array}{l}\text { Length } \\
\text { (bp) }\end{array}$ & $\begin{array}{l}\text { GC } \\
(\%)\end{array}$ & $\begin{array}{c}\text { Rate }^{\text {h }} \\
(\%)\end{array}$ & \\
\hline Promotor & 14202 & 5.51 & 20.98 & 31.26 & 11158 & 7.01 & 18.98 & 36.63 & 78.57 & 5297 & 14.78 & 13.94 & 24.94 & 46.42 & 37.30 \\
\hline 5'UTR & 3350 & 4.13 & 20.93 & 53.76 & 3002 & 4.61 & 19.64 & 62.57 & 89.61 & 1175 & 11.79 & 15.94 & 45.45 & 38.59 & 35.07 \\
\hline 3'UTR & 3776 & 5.38 & 18.18 & 30.93 & 3347 & 6.07 & 16.61 & 38.42 & 88.64 & 1930 & 10.52 & 12.79 & 21.02 & 57.13 & 51.11 \\
\hline CDS & 5737 & 18.79 & 22.03 & 70.77 & 4933 & 21.85 & 20.39 & 80.12 & 85.99 & 890 & 121.12 & 17.23 & 64.89 & 17.86 & 15.51 \\
\hline INTRON & 19232 & 7.64 & 19.11 & 37.89 & 15367 & 9.56 & 17.89 & 45.34 & 79.90 & 7779 & 18.90 & 14.18 & 31.66 & 49.52 & 40.45 \\
\hline INTERGENIC & 138796 & 13.67 & 20.42 & 43.19 & 49635 & 38.23 & 19.45 & 41.91 & 35.76 & 19955 & 95.08 & 13.85 & 37.33 & 37.81 & 14.38 \\
\hline Total & 179681 & 11.46 & 20.34 & 42.39 & 82694 & 24.90 & 19.09 & 43.90 & 46.02 & 35046 & 58.74 & 13.96 & 34.38 & 40.52 & 19.50 \\
\hline
\end{tabular}

${ }^{a}$ The overall SSRs were identified on the whole 10 chromosomes;

${ }^{b}$ The unique SSRs were SSRs with unique flanking sequences on the genome;

'Unique SSRs with polymorphisms were SSRs with length information results in ten or more than ten genomes, and PIC Value $\geq 0.5$;

${ }^{\mathrm{d}}$ Interval was calculated by number/Kb;

eSSR average length was expressed in base pairs (bp);

${ }^{f} \mathrm{GC}$ content was evaluated by percentages (\%);

${ }^{9}$ This rate was the percentage of unique SSRs against overall SSRs;

${ }^{\mathrm{h}}$ This rate was the percentage of unique SSRs with polymorphisms against unique SSRs;

'This rate was the percentage of unique SSRs with polymorphisms against overall SSRs;

'There were 179681 SSRs in total, due to the alternative splicing occurring in maize genome, and the same SSRs might be divided into different regions and double counting. 
Table 2 The proportion of SSRs with different types

\begin{tabular}{|c|c|c|c|c|c|c|c|c|c|c|c|c|c|c|}
\hline \multirow[t]{2}{*}{ Types } & \multirow[b]{2}{*}{$\begin{array}{c}\text { Repeat } \\
\text { units }\end{array}$} & \multicolumn{4}{|c|}{ Overall SSRs } & \multicolumn{4}{|c|}{ Unique SSRs } & \multicolumn{4}{|c|}{ Unique SSRs with polymorphisms } & \multirow[b]{2}{*}{$\begin{array}{c}\text { Rate } \\
(\%)\end{array}$} \\
\hline & & & Count & $\begin{array}{l}\text { Length } \\
\text { (bp) }\end{array}$ & $\begin{array}{l}\mathrm{GC} \\
\text { (\%) }\end{array}$ & $\begin{array}{l}\text { Rate } \\
(\%)\end{array}$ & Count & $\begin{array}{l}\text { Length } \\
\text { (bp) }\end{array}$ & $\begin{array}{l}\mathrm{GC} \\
\text { (\%) }\end{array}$ & $\begin{array}{c}\text { Rate } \\
(\%)\end{array}$ & Count & $\begin{array}{l}\text { Length } \\
\text { (bp) }\end{array}$ & $\begin{array}{l}\mathrm{GC} \\
(\%)\end{array}$ & \\
\hline \multirow[t]{7}{*}{ Perfect SSRs } & MNRs & 72258 & 11.70 & 53.21 & 40.21 & 35998 & 11.79 & 39.78 & 43.53 & 20632 & 11.20 & 30.33 & 58.87 & \\
\hline & DNRs & 53842 & 18.24 & 25.01 & 29.97 & 25510 & 20.38 & 22.49 & 30.85 & 9929 & 18.54 & 29.14 & 28.33 & \\
\hline & TNRs & 36726 & 17.52 & 49.12 & 20.44 & 12702 & 17.83 & 53.36 & 15.36 & 2557 & 19.04 & 47.18 & 7.30 & \\
\hline & TTRs & 2616 & 23.08 & 30.76 & 1.46 & 1784 & 23.45 & 34.81 & 2.16 & 685 & 24.05 & 31.43 & 1.95 & \\
\hline & PNRs & 800 & 28.37 & 41.10 & 0.45 & 482 & 28.05 & 41.36 & 0.58 & 225 & 27.31 & 43.09 & 0.64 & \\
\hline & HNRs & 449 & 32.54 & 51.24 & 0.25 & 199 & 32.38 & 57.14 & 0.24 & 101 & 31.96 & 56.41 & 0.29 & \\
\hline & Total $^{\mathrm{i}}$ & 166691 & 15.41 & 42.78 & 92.77 & 76675 & 16.08 & 34.92 & 92.72 & 34129 & 14.35 & 31.43 & 97.38 & \\
\hline Imperfect SSRs & - & 10841 & 91.66 & 47.45 & 6.03 & 4615 & 79.28 & 43.76 & 5.58 & 429 & 40.85 & 34.12 & 1.22 & \\
\hline Compound SSRs & - & 2149 & 43.48 & 33.44 & 1.20 & 1404 & 44.53 & 31.38 & 1.70 & 488 & 34.70 & 34.34 & 1.39 & \\
\hline Total & - & 179681 & 20.34 & 42.39 & 100.00 & 82694 & 20.09 & 36.74 & 100.00 & 35046 & 13.96 & 34.38 & 100.0 & \\
\hline
\end{tabular}

five types of perfect SSRs. Moreover, MNRs had the average length of $11.70 \mathrm{bp}$, which was the minimum of all SSRs. Furthermore, correlations between the number of observed SSRs and SSR length were taken into account. The results showed that the number of observed SSRs decreased with the increase of SSR length. For the perfect SSRs, the number of observed SSRs also sharply reduced with the increased motif repeats (Additional file 2).

The distribution of different SSR repeat types was surveyed as well. The major repeat types including MNRs, DNRs and TNRs accounted for almost $90 \%$ of the overall SSRs collectively. The distribution proportion of these SSRs in different genomic regions varied (Figure 1). Along with the increase of motif repeats, SSRs detected in all regions decreased except for CDS. In the CDS, MNRs shared only $4.71 \%$, which occupied almost half of the overall SSRs in any other regions. DNRs were analogous to MNRs in CDS with the minimum percentage of $6.72 \%$ and evenly distributed in intergenic, while UTRs and intron occupied $34.06 \%, 30.08 \%$ and $31.44 \%$ separately. In addition, TNRs predominated in CDS region, accounting for $88.58 \%$, but rarely distributed in any other regions.

\section{Different repeat units of perfect SSRs in maize}

Based on combinations of all four nucleotides, the canonical set of SSR motifs was represented by two different single nucleotides, four different duplets (AC, AG, AT, CG), 10 different triplets, 33 different quadruplets and 102 different quintuplet motifs [24]. All these expected SSR motifs could be represented in maize with variant forms of the same basic set or by their reverse complements. The frequencies of different motifs observed in different

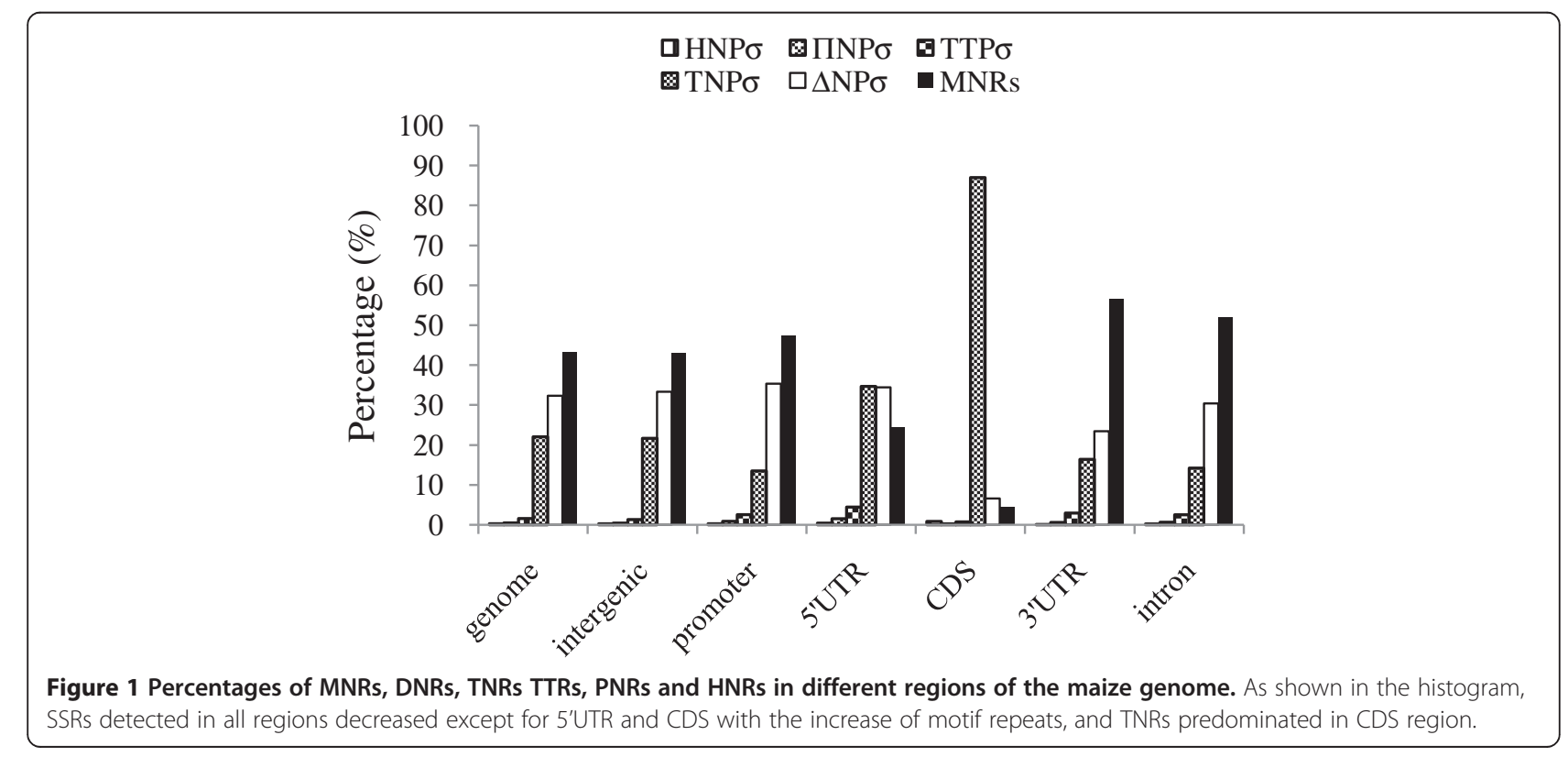


areas of the genome were variable. In general, $(\mathrm{C} / \mathrm{G}) \mathrm{n}$ was more abundant (24.44\%), followed by (A/T)n (19.94\%), (AG)n (16.06\%), and (AT)n (12.61\%), while the (GC)n motif was the least frequent $(1.28 \%)$ in maize genome. Of the trinucleotide motifs, (AGC)n was the most abundant (5.14\%), followed by (ACG)n (CCG)n (2.81\%), (2.61\%), (ATC)n (2.49\%), (AAG)n (2.48\%), (AAT)n (2.07\%), (ACC) n (2.04\%), (AGG)n (1.22\%), (AAC)n (0.85\%) and (ACT)n $(0.84 \%)$ (Table 3$)$. The remaining motifs were present in less than $10 \%$ of the total with too many combinations.

\section{The number and distribution of unique SSRs with polymorphism}

According to next-generation sequencing data from 345 maize inbred lines, there were 10,527.38 M reads, with average length of $186.02 \mathrm{bp}$. the sequencing depth of maize inbred line qi410 was the lowest of $0.07 x$, while the maize inbred line 478 had the highest sequencing depth of $40.25 \times$, with average sequencing depth of $2.89 x$ (Additional file 3). The length information of 346 maize inbred lines, including maize inbred line B73 reference genome, were analyzed by 82,694 SSRs with unique flanking sequences in this study. There were totally 58,946 SSRs with length information results in ten or more than ten genomes, accounting for $71.28 \%$ of SSRs with unique flanking sequences, while 55,621 of totally 58,946 SSRs had polymorphism with an average PIC value of 0.498 . However, there were 35,046 SSR loci with average PIC value $\geq 0.50$, accounting for $42.38 \%$ of SSRs with unique flanking sequences (Additional file 4). The distributions for overall SSRs, unique SSRs and unique SSR with polymorphism $(\mathrm{PIC} \geq 0.5)$ on maize chromosomes were shown in Figure $2(A, B, C)$. The distribution of SSRs on the chromosomes is non-uniform, and their density in subtelomeric regions tended to be higher than that in the regions nearing to the centromeres, which was in accordance with the distribution of genes in maize [25]. Additionally, the density of unique SSRs with polymorphism was much higher than that of published SSRs in MaizeGDB by comparing unique SSRs (Figure 2B), unique SSRs with polymorphism (Figure $2 \mathrm{C}$ ) and published SSRs in MaizeGDB (Figure 2D).

In general, with the increase of length differences in SSR sequences, the number of polymorphism SSRs was less. The length discrepancy of SSRs loci ranged from $1 \mathrm{bp}$ to $193 \mathrm{bp}$. The greatest length discrepancy of $4 \mathrm{bp}$ for SSR locus in different materials predominated with 7,580 , accounting for $21.63 \%$ of SSR polymorphism locus, while there were 35,928 SSR loci with the greatest length discrepancy $\geq 5 \mathrm{bp}$, accounting for $64.59 \%$ of SSR polymorphism locus. The polymorphism SSRs with more obvious length discrepancy in different genomes were much easier for detecting in experiments (Additional file 4).

For different regions in genome, as shown in Table 1, the unique polymorphism SSRs were most abundant in 3'UTR (57.13\%), followed in an order of intronic (49.52\%), promotor (46.42\%), 5'UTR (38.59\%), intergenic regions (37.81\%) and CDS (17.86\%). It was interesting to note

Table 3 The proportion of SSRs motifs in maize genome

\begin{tabular}{|c|c|c|c|c|c|c|c|c|c|}
\hline \multirow[t]{2}{*}{ Motif } & \multicolumn{3}{|c|}{ Total SSRs } & \multicolumn{3}{|c|}{ Unique SSRs } & \multicolumn{3}{|c|}{ Unique SSRs with polymorphisms } \\
\hline & Count & Length (bp) & Rate (\%) & Count & Length (bp) & Rate (\%) & Count & Length (bp) & Rate (\%) \\
\hline $\mathrm{A} / \mathrm{T}$ & 32462 & 10.96 & 19.94 & 22640 & 10.92 & 29.18 & 14221 & 10.87 & 42.94 \\
\hline$C / G$ & 39796 & 12.31 & 24.44 & 14597 & 13.14 & 18.81 & 6411 & 11.93 & 19.36 \\
\hline AT/TA & 20534 & 23.19 & 12.61 & 12169 & 13.26 & 15.68 & 3719 & 19.43 & 11.23 \\
\hline $\mathrm{CG} / \mathrm{GC}$ & 2085 & 12.56 & 1.28 & 1115 & 12.77 & 1.44 & 157 & 13.17 & 0.47 \\
\hline AC/GT/CA/TG & 5153 & 14.05 & 3.16 & 3129 & 14.45 & 4.03 & 1318 & 15.61 & 3.98 \\
\hline $\mathrm{AG} / \mathrm{CT} / \mathrm{TC} / \mathrm{GA}$ & 26070 & 15.63 & 16.01 & 10706 & 17.30 & 13.80 & 4735 & 18.83 & 14.30 \\
\hline AAC/GTT/TGT/ACA/CAA/TTG & 1389 & 17.93 & 0.85 & 1000 & 18.55 & 1.29 & 231 & 20.40 & 0.70 \\
\hline AAG/CTT/TTC/GAA/TCT/AGA & 4032 & 16.19 & 2.48 & 1263 & 16.90 & 1.63 & 316 & 18.13 & 0.95 \\
\hline AAT/ATT/TAA/TTA/TAT/ATA & 3378 & 23.13 & 2.07 & 1244 & 26.59 & 1.60 & 297 & 23.68 & 0.90 \\
\hline ACC/GGT/CAC/GTG/CCA/TGG & 3322 & 15.85 & 2.04 & 1193 & 16.18 & 1.54 & 165 & 17.58 & 0.50 \\
\hline ACG/CGT/GAC/GTC/CGA/TCG & 4244 & 17.23 & 2.61 & 1255 & 16.52 & 1.62 & 235 & 17.77 & 0.71 \\
\hline ACT/AGT/GTA/TAC/TAG/CTA & 1372 & 20.30 & 0.84 & 363 & 17.88 & 0.47 & 90 & 17.83 & 0.27 \\
\hline AGC/GCT/CTG/CAG/TGC/GCA & 8375 & 16.15 & 5.14 & 1773 & 16.33 & 2.28 & 364 & 17.27 & 1.10 \\
\hline AGG/CCT/GGA/TCC/CTC/GAG & 1981 & 16.98 & 1.22 & 1224 & 17.47 & 1.58 & 327 & 18.91 & 0.99 \\
\hline ATC/GAT/CAT/ATG/TCA/TGA & 4051 & 18.15 & 2.49 & 777 & 18.25 & 1.00 & 198 & 20.92 & 0.60 \\
\hline $\mathrm{CCG} / \mathrm{CGG} / \mathrm{GGC} / \mathrm{GCC} / \mathrm{GCG} / \mathrm{CGC}$ & 4582 & 16.38 & 2.81 & 3146 & 16.66 & 4.05 & 334 & 17.72 & 1.01 \\
\hline Total & 162826 & 15.15 & 100 & 77594 & 13.94 & 100 & 33118 & 14.01 & 100 \\
\hline
\end{tabular}




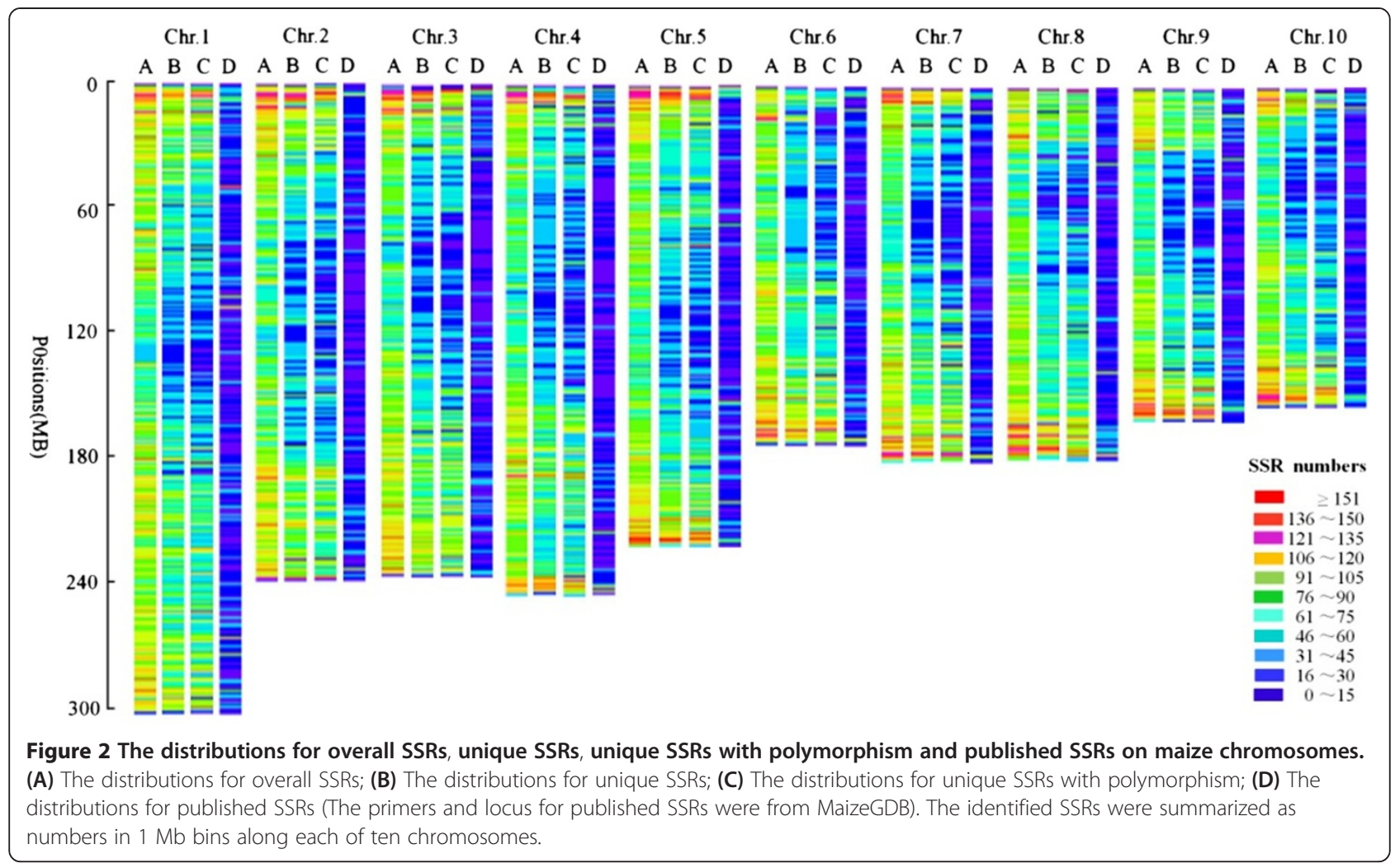

that the polymorphism in genic regions was greater than that in intergenic regions.

\section{Experimental validation for amplification efficiency and polymorphism of the developed SSR primers}

250 pairs of primers in different genomic regions covering all maize chromosomes were chosen for the experimental validation. The PIC value of SSR locus in 346 maize genomes ranged from 0 to 0.88 , with average PIC value of 0.49 . There were 102 SSR polymorphism locus with PIC value $<0.5$, with average PIC value of 0.24 , while 148 SSR polymorphism locus with $\mathrm{PIC}$ value $\geq 0.5$, with average PIC value of 0.66 . The PIC value of SSR locus in 11 elite maize inbred lines ranged from 0 to 0.89 , with average PIC value of 0.63 . There were 102 SSR polymorphism locus with PIC value $<0.5$, with average PIC value of 0.59 , while 148 SSR polymorphism locus with PIC value $\geq 0.5$, with average PIC value of 0.654 . The alleles detected per primer varied from 2 to 9 , with an average of 3.9. (Figure 3).

\section{Discussion}

The distribution of SSRs in maize

SSRs have been shown to be in both eukaryotic organisms and prokaryotes, with great differences across species in accumulating degree on varied regions of the genome [26]. In eukaryotes, one can expect to encounter at least one simple sequence stretch every $10 \mathrm{~kb}$ of DNA sequence
[26]. Based on the survey of human genome, one SSR was found to be every $6 \mathrm{~kb}$ on average [27]. The SSR frequency in maize was one in every $11.46 \mathrm{~Kb}$, which was lower than that in rice $(3.6 \mathrm{~Kb})[28]$. In general, minor difference was shown for SSR distribution in the same species or similar species. For instance, the distribution of SSRs was very similar with indica and japonica in general, the interval between two SSRs varied from $2.0 \mathrm{kbp}$ to $8.1 \mathrm{kbp}$, with highly dispersed in 5' UTR (interval was $2.1 \mathrm{kbp}$ and 2.0 $\mathrm{kbp}$, respectively) and lowly in CDS (interval was $8.1 \mathrm{kbp}$ and $7.7 \mathrm{kbp}$, respectively) [28]. However, the density of maize SSRs in different genomic regions was unbalanced, ranged from $5.51 \mathrm{kbp}$ (promotor) to $18.79 \mathrm{kbp}$ (CDS). The average GC content in maize SSR sequences (42.39\%) was much higher than that in rice genome (27.7\%). However, the average length of maize (20.34 bp) was almost equal to that of rice $(17.80 \mathrm{bp})$. For the SSR motif in maize genome, the proportion of MNRs, DNRs, and TNRs was around $40.21 \%, 29.97 \%$, and $20.44 \%$ respectively. However, the great discrepancy in the repeat unions of SSRs revealed that maize was rich in $\mathrm{C} / \mathrm{G}$ repeats for MNRs, AT repeats for DNRs, and AGC repeats for TNRs, but in rice, A/T, AG and AGG repeats were the most common for the three different types [28].

Intriguingly, the majority of SSRs which resided in CDS were TNRs. Similarly, more than $92 \%$ of the predicted SSR within coding sequences had repeat-unit sizes that were a multiple of three in a human cDNA database [29]. 


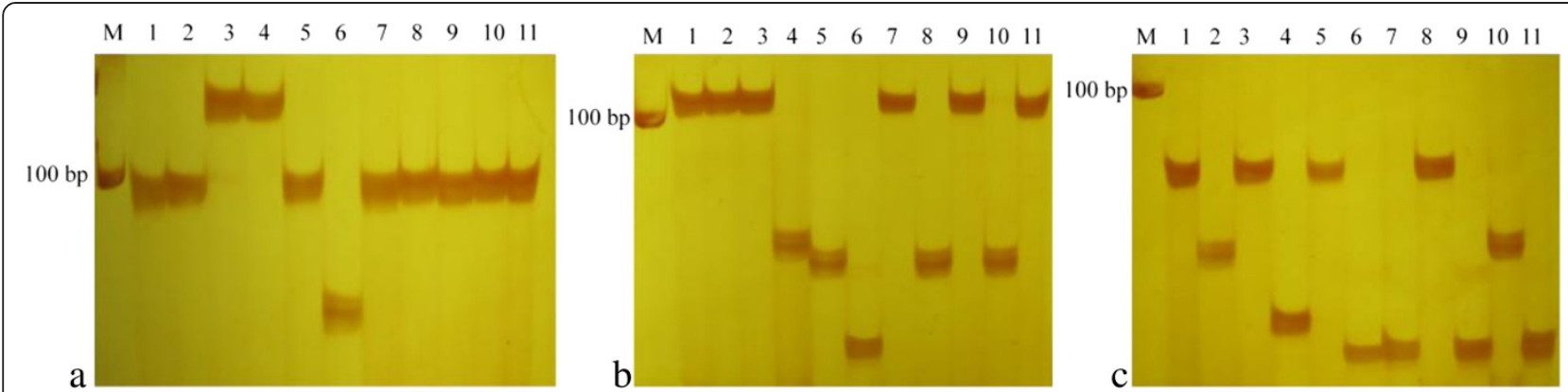

Figure 3 Experimental validation for three SSRs on chr5-206798023 (a), chr4-2629366 (b), and chr9-154777915 (c); PCR products from line1 to line11 are B73, Mo17, 91b30, R18, 1212, 583, 3237, R08, Huangzao Si, Zi330, and S37, respectively. (M: Marker DL2000).

The abundance of TNRs in CDS also supported that specific selection against frameshift mutations in coding regions [4,30]. TNRs had not generated frameshifts through expansion of triplet microsatellites, so that which would refrain from selective pressures in coding regions. However, non-triplet microsatellites had to be subject to greater purifying selection with the frameshifts mutations [30]. Therefore, mutation pressure contributed to the enrichment of TNRs in CDS. The strong reading frame and strand preferences were signs of effects of selection, against possible frame shift mutation.

\section{The polymorphism of SSRs in maize}

With the progress of next-generation sequencing technologies, the length of reads in the sequencing results increased gradually from $40 \mathrm{bp}$ initially to $200 \mathrm{bp}$ now. In the de novo sequencing data from maize inbred lines, reads with length more than $120 \mathrm{bp}$ accounted for $97.16 \%$. The average length of SSR in maize genome was $20.34 \mathrm{bp}$, with additional $50 \mathrm{bp}$ in the flanking sequence on both ends, so the average detected length was $70.34 \mathrm{bp}$. However, the detected SSRs with length lower than $120 \mathrm{bp}$ in SSRs with unique flanking sequences accounted for $97.35 \%$. Thus, most SSR locus could be detected via reads data from de novo sequencing, and different sequencing copies at the same loci could be used as repeats for enhancing data accuracy. Maize inbred line Mo17 was partly sequenced by Roche 454 technology, with average length of $400 \mathrm{bp}$, and 39,274 (47.49\%) SSR locus were detected in Mo17 genome. The detection rate of SSR locus in maize inbred lines with similar sequencing depth, such as zheng58, reached $46 \%$, which indicated that the detection rate depended on the sequencing depth. According to the length, $94.37 \%$ of SSR locus could be detected in the genome, but only $47.49 \%$ of SSR locus was detected in the genome of Mo17, a maize inbred line with the most results. SSR locus without detection results were mainly caused by the base discrepancy of flanking sequences.
SSRs were widely concerned and used as an ideal tool for deciphering genetic variability, not only due to the abundance within a genome, the random occurrence, but also the high degree of polymorphisms [27]. The analysis of SSR polymorphism locus in the sequencing data from 346 maize inbred lines revealed that SSR locus in maize genome had extensive polymorphism. According to the published researches on genetic diversity in maize inbred lines, the average PIC for SSR markers in different studies varied from 0.47 to 0.69 , with a mean value of 0.607 , which was in agreement with the results [31-41]. The results from experimental validation were slighter higher than that from 346 genomes. Unique SSR locus were selected for the alignment of SSR polymorphism locus via software, and each loci was only one value in each genome. However, there are always several bands in one material due to non-specific amplification during the experiment, which also increases SSR polymorphism locus in the detection. The developed SSR locus with great length discrepancy, high polymorphism and density might have a higher chance of polymorphism exhibition in populations. Therefore, SSRs primers are especially important and efficient for practical application value in genetic researches and molecular breeding.

Two points were noteworthy for unique SSRs with polymorphisms in maize. Firstly, the variation level in maize inbred lines was relatively high, and the polymorphism rate of model maize inbred lines between B73 and Mo17, accounting for $66.04 \%$, was even higher than that of rice subspecies, accounting for $51.80 \%$ [28]. Secondly, the polymorphism in intragenic regions of maize genome was higher than that in intergenic regions, while the opposite result was showed in rice.

Maize is a kind of species with high domestication and artificial selection, so it completely depends on humans for its survival, which leads to the fact that researchers cannot find the progenitor for maize in a long time. Hybrid maize breeding took full advantage of heterosis, and it was produced by inbred lines that originated from divergent heterotic groups. The greater the genetic 
variation in maize inbred lines was, the more obvious the heterosis phenomenon was. In the view of SSR polymorphism, the genetic variation in maize inbred lines was close to different rice subspecies, which also reflected that maize was a highly polymorphic species [42]. The results showed that the polymorphism of SSR was affected by artificial selection in the process of maize breeding. This selection aimed to function also directly showed that SSR polymorphism with $44.31 \%$ in intragenic regions of maize was higher than that with $37.81 \%$ in intergenic regions. Furthermore, the diversity of 462 SSRs in maize genome and its wild progenitor, teosinte were observed to reveal how the domestication bottlenecks and artificial selection shaped the amount and distribution of genetic variation in maize genome [43].

\section{Conclusions}

Our work provided insight into the non-random distribution spatterns and compositions of SSRs in different regions of maize genome, and also developed more polymorphic SSR markers using next-generation sequencing reads. The genome-wide SSRs polymorphism markers could be useful for genetic analysis and marker-assisted selection in breeding practice, and it was also proved to be high efficient for molecular marker development via next-generation sequencing reads.

\section{Methods}

\section{Maize genome sequence sources}

The genome sequences for maize B73 (Release ZmB73_ RefGen_v2) and Mo17 (454 pyrosequencing data) were downloaded from http://www.maizesequence.org/index. html and http://www.phytozome.net/maize.phprespectively. 5'UTR, coding determining sequences (CDS), 3'UTR, exon, intron and intergenic regions were provided by the annotation of B73 genome (ZmB73_5b_FGS, http:// $\mathrm{ftp}$.maizesequence.org/current/filtered-set/). The genomic DNA sequences of $2 \mathrm{~Kb}$ from upstream of star codon (ATG) were analyzed as promoters. The de novo sequencing data of 345 maize materials were downloaded from NCBI (http://www.ncbi.nlm.nih.gov/sra?term=SRA049859 and http://www.ncbi.nlm.nih.gov/sra?term=SRA051245), including 151 elite Chinese lines, 88 Ex-PVP lines, 50 improved maize lines, 23 maize landraces, 33 public US lines (Additional file 3).

\section{SSRs screening in maize reference genome B73}

Microsatellite search module (MISA), a SSRs motif scanning tool written in Perl (http://pgrc.ipk-gatersleben. $\mathrm{de} / \mathrm{misa} /$ ), was used for the identification and localization of perfect microsatellites, compound microsatellites and imperfect microsatellites which were interrupted by a certain number of bases [44]. The identified motifs were one to sixnucleotides in size, and the minimum repeat unit was defined as 10 for mononucleotides (MNRs), seven for di-nucleotides (DNRs), six for tri-nucleotides (TNRs), five for tetra-nucleotides (TTRs) and four for all the higher order motifs including penta-nucleotides (PNRs) and hexa-nucleotides (HNRs). Furthermore, the maximal number of interrupting base pairs in a compound microsatellite was $100 \mathrm{bp}$. The variation and reverse complement of each motif were categorized into the same groups.

\section{Identification of SSRs with unique flanking sequences}

The first 20 bp sequences of 5 bp in upstream of SSR loci were extracted by program written in Perl as the upstream primer of e-PCR, while the last 20 bp sequences of $5 \mathrm{bp}$ in downstream of SSR loci were extracted as the downstream prime after inverted repeats. These primer sequences with Bowtie software were aligned against to maize reference genome, allowing up to one mismatched, and then SSRs with unique flanking sequences were identified by program written in Perl [45].

\section{Variation of SSRs in $\mathbf{3 4 6}$ maize inbred lines}

Taking the maize reference genome sequence and reads of the de novo sequencing data from 345 maize inbred lines as the template, the sequences around SSRs with unique flanking sequences were aligned against via Bowtie to extract the length information about SSR loci by program written in Perl. The allelic diversity of each SSR locus was assessed by the polymorphism information content (PIC), which is defined as $\mathrm{PIC}_{i}=1-\sum_{j=1}^{n} p_{i j}^{2}$, where $\mathrm{p}_{i j}$ is the frequency of the $j$ th pattern for the $i$ th markers. Furthermore, the length of polymorphism SSRs was investigated in the reference genome [46].

\section{PCR-based primer design}

The unique hits were selected for primer design. The sequences include SSR motif and two $100 \mathrm{bp}$ flanking sequences on each side of the repeat were used for automatically primer designed by Primer3 [47] through following parameters: primer length range from $20 \mathrm{nt}$ to $28 \mathrm{nt}$, with optimum $23 \mathrm{nt}$; melting temperature (Tm) of $60^{\circ} \mathrm{C}$ to $65^{\circ} \mathrm{C}$, with optimum temperature of $63^{\circ} \mathrm{C}$, and primer pairs must have a similar Tm value with GC content around 50\%, ranging from $30 \%$ to $70 \%$; the expected product size of $80 \mathrm{bp}$ to $200 \mathrm{bp}$ perfect ending with G- or C-rich at the 3' end.

\section{Experimental validation for amplification efficiency and polymorphism of the developed SSR primers}

250 pairs of primers covering all maize chromosomes synthetized by Shanghai Invitrogen Co., Ltd were used 
for the validation experiment. The materials selected in this experiment were elite inbred lines cataloged to four major heterotic groups in China. The DNA template were B73, Mo17, 91b30, R18, 1212, 583, 3237, R08, Huangzao Si, Zi330, and S37. Genomic DNA was extracted from two weeks old seedlings employing the modification of a CTAB (cetyltrimethylammonium bromide) DNA extraction protocol. PCR was performed in a reaction mixture of $15 \mu \mathrm{L}$, containing $50 \mathrm{ng}$ total genomic DNA for template, $1.5 \mu \mathrm{L}$ $10 \times$ buffer $\left(\mathrm{Mg}^{2+}\right), 2.0 \mu \mathrm{L}$ dNTP $(2.5 \mathrm{mM}), 100 \mathrm{nM}$ of each SSR-primer, $2 \mathrm{U}$ Taq polymerase, and $\mathrm{ddd}_{2} \mathrm{O}$. The $\mathrm{C} 1000$ thermal cycler (Bio-rad, Inc., Hercules, CA) was used for amplification with the following protocol: an initial denaturation for 3 minutes at $95^{\circ} \mathrm{C}, 35$ cycles of denaturation for 30s at $95^{\circ} \mathrm{C}$, annealing for $90 \mathrm{~s}$ at $55^{\circ} \mathrm{C}$, and an extension for $90 \mathrm{~s}$ at $72^{\circ} \mathrm{C}$; and a final extension for 10 minutes at $72^{\circ} \mathrm{C}$. PCR products were electrophoresed on $6.0 \%$ polyacrylamide gel. The PIC value for each SSR marker was calculated via the formula previously described.

\section{Additional files}

Additional file 1: The distribution of SSRs on chromosome of maize
genome.
Additional file 2: The length of SSRs with different types.
Additional file 3: 346 maize materials, including their name,
category, sequencing depth and average read length.
Additional file 4: 82694 SSRs markers, including their locus, motif
and PIC.

\section{Competing interests}

The authors declare that they have no competing interests.

\section{Authors' contributions}

QJT performed bioinformatic analysis, primer design and tested SSR markers. $J \mathrm{~L}$ designed, coordinated the study and preparing the manuscript. All authors read and approved the final manuscript.

\section{Acknowledgments}

The financial supported this research was provided by the National Basic Research Program (the "973" project, 2014CB138203), National Natural Science Foundation (31101161) and Sichuan Youth \& technology foundation (08ZQ026-011).

Received: 12 September 2013 Accepted: 12 September 2013 Published: 7 October 2013

\section{References}

1. Morgante M, Hanafey M, Powell W: Microsatellites are preferentially associated with nonrepetitive DNA in plant genomes. Nature genetics 2002, 30(2):194-200.

2. Kashi Y, King DG: Simple sequence repeats as advantageous mutators in evolution. Trends Genet 2006, 22(5):253-259.

3. Li YC, Korol AB, Fahima T, Beiles A, Nevo E: Microsatellites: genomic distribution, putative functions and mutational mechanisms: a review. Mol Ecol 2002, 11(12):2453-2465.

4. Li YC, Korol AB, Fahima T, Nevo E: Microsatellites within genes: structure, function, and evolution. Mol Biol and Evol 2004, 21(6):991-1007.

5. Varshney RK, Graner A, Sorrells ME: Genic microsatellite markers in plants: features and applications. TRENDS in Biotechnology 2005, 23(1):48-55.
6. Ramu P, Kassahun B, Senthilvel S, Ashok Kumar C, Jayashree B, Folkertsma R, Reddy LA, Kuruvinashetti M, Haussmann BIG, Hash C: Exploiting rice-sorghum synteny for targeted development of EST-SSRs to enrich the sorghum genetic linkage map. TAG Theor App/ Genet 2009, 119(7):1193-1204.

7. Powell W, Morgante M, Andre C, Hanafey M, Vogel J, Tingey S, Rafalski A: The comparison of RFLP, RAPD, AFLP and SSR (microsatellite) markers for germplasm analysis. Mol Breed 1996, 2(3):225-238.

8. Wang Y, Georgi LL, Zhebentyayeva TN, Reighard GL, Scorza R, Abbott AG: High-throughput targeted SSR marker development in peach (Prunus persica). Genome 2002, 45(2):319-328.

9. Mrázek J: Analysis of distribution indicates diverse functions of simple sequence repeats in Mycoplasma genomes. Mol Biol and Evol 2006, 23(7):1370-1385.

10. Verstrepen KJ, Jansen A, Lewitter F, Fink GR: Intragenic tandem repeats generate functional variability. Nat Genet 2005, 37(9):986-990.

11. Brown LY, Brown SA: Alanine tracts: the expanding story of human illness and trinucleotide repeats. Trends Genet 2004, 20(1):51-58.

12. Fujimori S, Washio T, Higo K, Ohtomo Y, Murakami K, Matsubara K, Kawai J, Carninci P, Hayashizaki Y, Kikuchi S: A novel feature of microsatellites in plants: a distribution gradient along the direction of transcription. FEBS letters 2003, 554(1-2):17-22.

13. Bao J, Corke H, Sun M: Microsatellites in starch-synthesizing genes in relation to starch physicochemical properties in waxy rice (Oryza sativa L.) TAG Theor App/ Genet 2002, 105(6):898-905.

14. Dresselhaus T, Cordts S, Heuer S, Sauter M, Lörz H, Kranz E: Novel ribosomal genes from maize are differentially expressed in the zygotic and somatic cell cycles. Mol Gen Genet MGG 1999, 261(2):416-427.

15. Xiong L-W, Wang Q, Qiu G-F: Large-scale isolation of microsatellites from Chinese mitten crab eriocheir sinensis via a solexa genomic survey. International journal of molecular sciences 2012, 13(12):16333-16345.

16. An HS, Lee JW: Development of microsatellite markers for the Korean mussel, mytilus coruscus (Mytilidae) using next-generation sequencing. International journal of molecular sciences 2012, 13(8):10583-10593.

17. Ji P, Zhang Y, Li C, Zhao Z, Wang J, Li J, Xu P, Sun X: High throughput mining and characterization of microsatellites from common carp genome. International journal of molecular sciences 2012, 13(8):9798-9807.

18. Luo W, Nie Z, Zhan F, Wei J, Wang W, Gao Z: Rapid development of microsatellite markers for the endangered fish schizothorax biddulphi (Günther) using next generation sequencing and cross-species amplification. International journal of molecular sciences 2012, 13(11):14946-14955.

19. Schnable PS, Ware D, Fulton RS, Stein JC, Wei F, Pasternak S, Liang C, Zhang J, Fulton L, Graves TA: The B73 maize genome: complexity, diversity, and dynamics. science 2009, 326(5956):1112-1115.

20. Baucom RS, Estill JC, Chaparro C, Upshaw N, Jogi A, Deragon JM, Westerman RP, SanMiguel PJ, Bennetzen JL: Exceptional diversity, nonrandom distribution, and rapid evolution of retroelements in the B73 maize genome. PLoS Genet 2009, 5(11):e1000732.

21. Yang L, Bennetzen JL: Distribution, diversity, evolution, and survival of Helitrons in the maize genome. Proc Natl Acad Sci 2009, 106(47):19922-19927.

22. Jiao Y, Zhao H, Ren L, Song W, Zeng B, Guo J, Wang B, Liu Z, Chen J, Li W, et al: Genome-wide genetic changes during modern breeding of maize. Nat Genet 2012, 44(7):812-815.

23. Chia JM, Song C, Bradbury PJ, Costich D, de Leon N, Doebley J, Elshire RJ, Gaut B, Geller L, Glaubitz JC, et al: Maize HapMap2 identifies extant variation from a genome in flux. Nat Genet 2012, 44(7):803-807.

24. La Rota M, Kantety R, Yu JK, Sorrells M: Nonrandom distribution and frequencies of genomic and EST-derived microsatellite markers in rice, wheat, and barley. BMC genomics 2005, 6(1):23.

25. Soderlund C, Descour A, Kudrna D, Bomhoff M, Boyd L, Currie J, Angelova A, Collura K, Wissotski M, Ashley E: Sequencing, mapping, and analysis of 27,455 maize full-length cDNAs. PLoS Genet 2009, 5(11):e1000740.

26. Tautz D: Hypervariabflity of simple sequences as a general source for polymorphic DNA markers. Nucleic Acids Res 1989, 17(16):6463-6471.

27. Beckmann JS, Weber JL: Survey of human and rat microsatellites. Genomics 1992, 12(4):627-631.

28. Zhang Z, Deng Y, Tan J, Hu S, Yu J, Xue Q: A genome-wide microsatellite polymorphism database for the indica and japonica rice. DNA Res 2007, 14(1):37-45

29. Wren JD, Forgacs E, Fondon JW, Pertsemlidis A, Cheng SY, Gallardo T, Williams R, Shohet RV, Minna JD, Garner HR: Repeat polymorphisms within 
gene regions: phenotypic and evolutionary implications. Am J Hum Genet 2000, 67(2):345-356.

30. Metzgar $D$, Bytof J, Wills $C$ : Selection against frameshift mutations limits microsatellite expansion in coding DNA. Genome Res 2000, 10(1):72-80.

31. Yang X, Xu Y, Shah T, Li H, Han Z, Li J, Yan J: Comparison of SSRs and SNPs in assessment of genetic relatedness in maize. Genetica 2011, 139(8):1045-1054.

32. Adeyemo O, Menkir A, Melaku G, Omidiji O: Genetic diversity assessment and relationship among tropical-yellow endosperm maize inbred lines using SSR markers. Maydica 2011, 56(1):43.

33. Smith J, Chin E, Shu H, Smith O, Wall S, Senior M, Mitchell S, Kresovich S, Ziegle J: An evaluation of the utility of SSR loci as molecular markers in maize (Zea mays L.): comparisons with data from RFLPs and pedigree. TAG Theor Appl Genet 1997, 95(1):163-173.

34. Senior M, Murphy J, Stuber C, Goodman M: Utility of SSRs for determining genetic similarities an relationships in maize using an agarose gel system. Crop Sci 1998, 38(4):1088-1098.

35. Enoki H, Sato H, Koinuma K: SSR analysis of genetic diversity among maize inbred lines adapted to cold regions of Japan. TAG Theor Appl Genet 2002, 104(8):1270-1277.

36. Li Y, Du J, Wang T, Shi Y, Song Y, Jia J: Genetic diversity and relationships among Chinese maize inbred lines revealed by SSR markers. Maydica 2002, 47(2):93-102.

37. Wang R, Yu Y, Zhao J, Shi Y, Song Y, Wang T, Li Y: Population structure and linkage disequilibrium of a mini core set of maize inbred lines in China. TAG Theor Appl Genet 2008, 117(7):1141-1153.

38. Legesse W, Legesse WB, MYBURG AA, PIXLEY VK, BOTHA MA: Genetic diversity of African maize inbred lines revealed by SSR markers. Hereditas 2007, 144(1):10-17.

39. Yuan L, Fu J, Warburton M, Li X, Zhang S, Khairallah M, Liu X, Peng Z, Li L: Comparison of genetic diversity among maize inbred lines based on RFLPs, SSRs, AFLPs and RAPDs]. Yi chuan xue bao = Acta genetica Sinica 2000, 27(8):725.

40. Xia X, Reif J, Hoisington D, Melchinger A, Frisch M, Warburton M: Genetic diversity among CIMMYT maize inbred lines investigated with SSR markers: I. Lowland tropical maize. CROP SCIENCE-MADISON-2004, 44:2230-2237.

41. Patto MCV, Satovic Z, Pego S, Fevereiro P: Assessing the genetic diversity of Portuguese maize germplasm using microsatellite markers. Euphytica 2004, 137(1):63-72.

42. Buckler ES, Gaut BS, McMullen MD: Molecular and functional diversity of maize. Curr Opin Plant Biol 2006, 9(2):172-176.

43. Vigouroux Y, Mitchell S, Matsuoka Y, Hamblin M, Kresovich S, Smith JSC, Jaqueth J, Smith OS, Doebley J: An analysis of genetic diversity across the maize genome using microsatellites. Genetics 2005, 169(3):1617-1630.

44. Thiel T, Michalek W, Varshney R, Graner A: Exploiting EST databases for the development and characterization of gene-derived SSR-markers in barley (Hordeum vulgare L.). TAG Theor Appl Genet 2003, 106(3):411-422.

45. Langmead B, Trapnell C, Pop M, Salzberg SL: Ultrafast and memoryefficient alignment of short DNA sequences to the human genome. Genome Biol 2009, 10(3):R25.

46. Anderson JA, Churchill GA, Autrique JE, Tanksley SD, Sorrells ME: Optimizing parental selection for genetic linkage maps. Genome 1993, 36(1):181-186.

47. Rozen S, Skaletsky H: Primer3 on the WWW for general users and for biologist programmers. Methods Mol Biol 2000, 132(3):365-386.

\section{doi:10.1186/1756-0500-6-403}

Cite this article as: $\mathrm{Qu}$ and Liu: A genome-wide analysis of simple sequence repeats in maize and the development of polymorphism markers from next-generation sequence data. BMC Research Notes 2013 6:403.

\section{Submit your next manuscript to BioMed Central and take full advantage of:}

- Convenient online submission

- Thorough peer review

- No space constraints or color figure charges

- Immediate publication on acceptance

- Inclusion in PubMed, CAS, Scopus and Google Scholar

- Research which is freely available for redistribution

Submit your manuscript at www.biomedcentral.com/submit
Ciomed Central 